\title{
FENÓMENO EL NIÑO Y DESASTRES NATURALES: INTERVENCIONES EN SALUD PÚBLICA PARA LA PREPARACIÓN Y RESPUESTA
}

\author{
Gisely Hijar ${ }^{1, a}$, Catherine Bonilla ${ }^{1, b}$, Cesar V. Munayco ${ }^{2, c}$, Ericson L. Gutierrez ${ }^{1,3, d}$, \\ Willy Ramos ${ }^{4, d}$
}

\begin{abstract}
RESUMEN
El presente artículo realiza una revisión de las intervenciones en salud pública para la preparación y respuesta ante desastres naturales en el contexto del fenómeno El Niño (FEN) a partir de revisiones sistemáticas y una revisión de revisiones con énfasis en las enfermedades transmitidas por vectores, transmitidas por el agua, malnutrición, estrés térmico, sequías, enfermedades asociadas a inundaciones, problemas de salud mental, vulnerabilidad de la infraestructura física de los servicios de salud, así como de las políticas a largo plazo orientadas a proteger a la población en estos casos. Se identificaron intervenciones ambientales, como el control de vectores, quimioprofilaxis, vacunación y tratamiento intradomiciliario de agua. Si bien estos hallazgos se basan principalmente en revisiones sistemáticas, es necesario evaluar el beneficio de estas intervenciones en la población, de acuerdo con la realidad de cada región.
\end{abstract}

Palabras clave: El Niño Oscilación del Sur; Desastres; Política social (fuente DeCS BIREME).

\section{EL NIÑO PHENOMENON AND NATURAL DISASTERS: PUBLIC HEALTH INTERVENTIONS FOR DISASTER PREPAREDNESS AND RESPONSE}

\begin{abstract}
This article reviews public health interventions for preparedness and response to natural disasters within the context of EI Niño phenomenon using systematic reviews and a review of revisions with emphasis on vector-borne diseases, waterborne diseases, malnutrition, heat stress, drought, flood-associated diseases, mental health problems, vulnerability of the physical health-system infrastructure, as well as long-term policies aimed at protecting the populations of these cases. Environmental interventions were identified, including vector control, chemoprophylaxis, immunization, and intradomiciliary water treatment. While these finds are based primarily on systematic reviews, it is necessary to evaluate the benefit of these interventions within the population, according to the context of each region.
\end{abstract}

Key words: El Nino-Southern Oscillation; Disasters; Public policy (source: MeSH NLM).

\section{INTRODUCCIÓN}

Un agente clave para la determinación del clima mundial es el océano. El océano y la atmósfera conforman un sistema acoplado, intercambiando energía mecánica y termodinámica. El océano participa en el calentamiento de la atmósfera proveyendo casi el $50 \%$ de su energía, de esta manera, cuando la temperatura superficial del mar es superior a la de la atmósfera, el mar se evapora $y$ transmite energía en forma de vapor de agua ${ }^{(1-3)}$.

El fenómeno El Niño (FEN) es un fenómeno océanoatmosférico que surge como manifestación de la elevación de la temperatura de la superficie del mar, debido al ingreso de grandes cantidades de aguas cálidas del pacifico occidental hacia el pacifico ecuatorial, que se produce por alteraciones de la presión atmosférica. Se declara un FEN cuando existe un incremento de la temperatura superficial promedio del mar en $0,5^{\circ} \mathrm{C}$, o más, por al menos 5 meses consecutivos ${ }^{(4-6)}$.

EI FEN constituye el más importante ciclo climático que contribuye a la variabilidad climática anual y comprende los cambios observados en la temperatura superficial del mar en el Pacífico ecuatorial central, así como los cambios de la presión atmosférica en el Pacífico,

\footnotetext{
Unidad de Análisis y Generación de Evidencias en Salud Pública. Centro Nacional de Salud Pública. Instituto Nacional de Salud. Lima, Perú. Department of Preventive Medicine and Biostatistics, Uniformed Services University of the Health Sciences. Bethesda, EE.UU.

Facultad de Medicina, Universidad de San Martín de Porres. Lima, Perú.

Instituto de Investigaciones Clínicas, Universidad Nacional Mayor de San Marcos. Lima, Perú.

Bióloga, magíster en Epidemiología; ${ }^{\mathrm{b}}$ nutricionista, magíster en Nutrición; ${ }^{\mathrm{c}}$ médico epidemiólogo y salubrista; ${ }^{\mathrm{d}}$ médico especialista en Gestión en Salud, d médico magíster en Epidemiología.

Recibido: 10/02/2016 Aprobado: 20/04/2016
}

Citar como: Hijar G, Bonilla C, Munayco CV, Gutierrez EL, Ramos W. Fenómeno El Niño y desastres naturales: intervenciones en salud pública para la preparación y respuesta. Rev Peru Med Exp Salud Publica. 2016;33(2):300-10. doi: 10.17843/rpmesp.2016.332.2205. doi: 10.17843/rpmesp.2016.332.2205 
desde Australia (Darwin) hasta Tahití (Pacífico tropical central-oriental) ${ }^{(6)}$. El Niño y La Niña formarían parte de un mismo fenómeno conocido como Oscilación del Sur, donde El Niño constituiría la fase caliente y La Niña la fase fría ${ }^{(7)}$. Durante el FEN, el océano transmite energía a la atmósfera y, como consecuencia, aumenta la temperatura; un ejemplo de esto lo constituye el FEN 1997-1998 en el que la temperatura ambiental de la ciudad de Lima se incrementó $5{ }^{\circ} \mathrm{C}$ por encima de lo normal ${ }^{(4-6)}$. Las modificaciones climáticas producidas por el FEN tienen efectos en la economía y en actividades productivas de los países, principalmente en la pesca, en la agricultura y ganadería (Tabla 1).

EI FEN ejerce sus efectos en la salud de forma directa o indirecta. De forma directa al modificar condiciones climáticas como la temperatura y humedad ambiental ${ }^{(6)} y$ de forma indirecta a través de la ocurrencia de desastres naturales ${ }^{(8,9)}$.

El objetivo de la presente revisión es identificar las intervenciones en salud pública efectivas para la preparación y respuesta ante desastres naturales en el contexto del fenómeno El Niño (FEN).

\section{EL FEN Y LOS DESASTRES NATURALES}

Los desastres son eventos generalmente repentinos e imprevistos que ocasionan daños, pérdidas y paralización temporal de actividades en cierta área, y que afectan a una parte importante de la población (10-13). Diversas condiciones hidrometeorológicas, geológicas y climáticas favorecen la ocurrencia de desastres naturales, los cuales se asocian a pérdida de vidas y

Tabla 1. Efectos del fenómeno El Niño en las actividades productivas y la economía de los países

\begin{tabular}{|c|c|}
\hline $\begin{array}{l}\text { Actividad o } \\
\text { sector afectado }\end{array}$ & Efectos del fenómeno El Niño \\
\hline Pesca & $\begin{array}{l}\text { - Principal actividad económica afectada. } \\
\text { - Migración de las especies marinas a } \\
\text { áreas de menor temperatura. } \\
\text { - Afectación de la pesca industrial por } \\
\text { escasez de anchoveta. } \\
\text { - Caída en la producción de harina de } \\
\text { pescado. }\end{array}$ \\
\hline Agricultura & $\begin{array}{l}\text { - Afectada por el incremento de la } \\
\text { temperatura y humedad así como por las } \\
\text { inundaciones. } \\
\text { - Reducción de las cosechas de tubérculos } \\
\text { y frutas. }\end{array}$ \\
\hline Ganadería & $\begin{array}{l}\text { - Reducción de disp onibilidad de insumos } \\
\text { ganaderos e incremento de sus costos. } \\
\text { - Baja producción de carne y leche. }\end{array}$ \\
\hline Exportaciones & $\begin{array}{l}\text { - Reducción por el impacto del FEN sobre } \\
\text { las actividades pesquera y agrícola. }\end{array}$ \\
\hline Energía & $\begin{array}{l}\text { - Daños en las centrales hidroeléctricas } \\
\text { por el excesivo caudal de los ríos. }\end{array}$ \\
\hline Transporte & $\begin{array}{l}\text { - Impacto por los efectos de los desastres } \\
\text { que destruyen carreteras y puentes. }\end{array}$ \\
\hline
\end{tabular}

lesiones, afectación de las condiciones de vida de las poblaciones (hacinamiento, intemperie), pérdida de bienes, daño e interrupción de los servicios básicos, daños en la infraestructura, destrucción del hábitat de animales (roedores), entre otros ${ }^{(14)}$.

El FEN incrementa la probabilidad de fenómenos meteorológicos extremos y ha sido asociado a desastres naturales como lluvias torrenciales, tormentas, incremento de la descarga de los ríos, inundaciones, formación y reactivación de quebradas, huracanes, tifones, ciclones y sequías ${ }^{(8,9)}$. Existen estudios que muestran la existencia de correlación entre las anomalías de la temperatura superficial del mar y el número de personas afectadas por desastres naturales ${ }^{(8)}$, esta correlación es más fuerte para el sur de Asia, que es donde se presentaron más de la mitad de personas afectadas en el período 1964-93.

Una de las características del FEN del año 1982-1983 fue la gran extensión del planeta que se vio afectada por sus efectos. En el Perú existió un notable incremento en la temperatura superficial del mar y del ambiente, así como fuertes lluvias a lo largo de varios meses (principalmente en Tumbes, Piura y Lambayeque), también ocurrieron grandes descargas de los ríos, lo cual se asoció a inundaciones así como a la reactivación y formación de quebradas (huaycos) ${ }^{(15-18)}$. Los eventos del FEN ocurridos en los años 1997 y 1998 son comparables en intensidad a los ocurridos en los años 1997-1998; sin embargo, los daños y pérdidas ocasionados en este último periodo fueron mayores.

De esta manera, se estima que el costo de los daños producidos por el FEN del 1982-1983 fue de 1000 millones de dólares; y los producidos por el FEN de 19971998 ascendieron a 3498 millones de dólares, según cifras del Banco de Desarrollo de América Latina ${ }^{(16)}$.

Los desastres naturales también tienen un efecto significativo en las tasas de reproducción y mortalidad de insectos o roedores ${ }^{(13)}$. Por otro lado, cuando ocurre un desastre natural, se generan diversas condiciones como el colapso de los servicios de agua y alcantarillado, exposición de la población a condiciones ambientales (humedad, agua estancada y/o polvo ambiental), interrupción de vías de comunicación, entre otros; los cuales, sumados a los cambios en la temperatura ambiental, pueden dar lugar a que se produzcan brotes epidémicos ${ }^{(14,19)}$. El FEN ha sido asociado con brotes epidémicos de malaria, dengue, influenza estacional, meningitis meningocócica, leptospirosis, cólera, fiebre tifoidea, shigelosis, peste bubónica, infecciones de la piel y conjuntivitis. Las enfermedades asociadas al FEN (20-55) se muestran en la tabla 2.

En nuestro país, el FEN 1982-1983 trajo consigo un incremento en el número de casos de malaria, infecciones 
Tabla 2. Enfermedades relacionadas al fenómeno El Niño y/o desastres naturales

\begin{tabular}{|c|}
\hline Grupo de enfermedades \\
\hline Transmitidas por vectores \\
\hline Dengue ${ }^{(20-28)}$ \\
\hline Malaria ${ }^{(29-32)}$ \\
\hline Leishmaniosis ${ }^{(33)}$ \\
\hline Bartonelosis ${ }^{(34)}$ \\
\hline Enfermedades zoonóticas \\
\hline Hantavirus ${ }^{(35)}$ \\
\hline Leptospirosis ${ }^{(36,37)}$ \\
\hline Peste ${ }^{(37)}$ \\
\hline Transmitidas por el agua y/o alimentos contaminados \\
\hline Cólera ${ }^{(38-42)}$ \\
\hline Fiebre tifoidea ${ }^{(37)}$ \\
\hline Shigelosis ${ }^{(38)}$ \\
\hline Infecciones del sistema nervioso \\
\hline Meningitis meningocócica ${ }^{(43)}$ \\
\hline Enfermedades de la piel y mucosas \\
\hline Verrugas virales, rosácea ${ }^{(44)}$ \\
\hline Conjuntivitis ${ }^{(37)}$ \\
\hline Enfermedades respiratorias \\
\hline IRA ${ }^{(37)}$ \\
\hline Influenza ${ }^{(45,46)}$ \\
\hline Problemas y trastornos de la salud mental \\
\hline Ansiedad, depresión, estrés postraumático, suicidio ${ }^{(47-55)}$ \\
\hline
\end{tabular}

respiratorias agudas y fiebre tifoidea. Por esos años no existió una adecuada gestión de los datos obtenidos, por lo que se perdió información de gran valor.

En el FEN de los años 1997-1998 existieron brotes epidémicos de cólera (focalizados en Tumbes, Lambayeque, Ica, Lima y Ucayali) y peste bubónica, también se apreció un incremento del número de casos notificados de malaria, dengue, infecciones respiratorias agudas, de las infecciones de la piel y conjuntivitis en las zonas afectadas por desastres ${ }^{(7,37)}$.

La infraestructura física de los servicios de salud se afecta gravemente por las manifestaciones del FEN, que produce daños por inundaciones, deterioro de los equipos y mobiliarios, efectos en techos, paredes, ventanas, materiales e insumos; y bloqueo y sedimentación de los sistemas de desagüe y alcantarillado, suministro y abastecimiento de agua potable, electricidad, gas y combustibles ${ }^{(37)}$.

\section{INTERVENCIONES DE SALUD PÚBLICA FRENTE A DESASTRES NATURALES}

La clave en la gestión de las actividades para afrontar los desastres naturales producto del cambio climático o fenómenos climáticos como el FEN, debe considerar las tres fases del "ciclo de los desastres": prevención, adaptación y mitigación o respuesta ${ }^{(56,57)}$. Las intervenciones en salud pública deben centrarse en estos tres puntos claves, pero, lamentablemente, las intervenciones, en general, se han enfocado principalmente a la mitigación, dejando de lado a la preparación y adaptación ${ }^{58,59)}$.

\section{FASE DE PREVENCIÓN}

Comprende un conjunto de acciones cuyo objeto es impedir o evitar que sucesos naturales o generados por la actividad humana, causen desastres. Dentro de la fase de prevención se encuentra el análisis de vulnerabilidades, que tiene la finalidad de identificar potenciales factores de riesgo, áreas y poblaciones en riesgo, con la finalidad de enfrentarlos con antelación al fenómeno climático o desastre natural ${ }^{57,58)}$.

\section{FASE DE ADAPTACIÓN}

Esta fase comprende un conjunto de medidas cuyo objetivo es organizar y estructurar la respuesta de la comunidad a las condiciones adversas; educar y capacitar a la población, con el objeto de facilitar las acciones para un efectivo y oportuno aviso, control, evacuación. Esta fase tiene por objetivo crear ciudades y sistemas de salud resilientes frente a los desastres, es decir, prepararlos para a) reducir el riesgo de desastres; b) adecuación del medioambiente, y c) planificación urbana ${ }^{(57,59)}$. Un estudio realizado en Nicaragua ${ }^{(59)}$, sugiere que las medidas de adaptación y mitigación frente a desastres naturales deben ser incluidas en las políticas públicas, para mejorar significativamente la eficiencia y eficacia de la respuesta frente a desastres naturales.

El papel de los profesionales de salud asistenciales en los desastres naturales ha cambiado considerablemente en las últimas tres décadas, se ha comenzado a trabajar en la preparación con el fin de mejorar la asistencia humanitaria brindada a la población. Los programas de capacitación y entrenamiento para el personal de salud deben incluirse en las políticas del sector Salud. Una revisión sugiere que el entrenamiento y la preparación de las enfermeras es vital para poder responder adecuadamente a los desastres naturales ${ }^{(60)}$.

\section{FASE DE MITIGACIÓN O RESPUESTA}

En esta fase se realiza la respuesta planificada y oportuna, para que una amenaza no se convierta en desastre, se lleva a cabo generalmente después de ocurrido el evento adverso, y requiere la realización de un conjunto de actividades que deben ser integrales, 
multifactoriales y multisectoriales, con el fin de salvar vidas, reducir sufrimientos, pérdidas económicas y daños ambientales. Esta fase permite disminuir el impacto de los desastres naturales, en ese sentido, la respuesta inmediata, organizada y efectiva es vital ${ }^{(59,61)}$.

Un reporte de la Organización Panamericana de Salud (OPS), sobre las repercusiones sanitarias del FEN $1997-$ 1998, indica que en América Latina, prácticamente todos los países afectados, establecieron planes específicos de contingencia y prepararon proyectos orientados a mitigar y a responder de la mejor manera posible los efectos provocados por los desastres atribuibles al FEN. Según el reporte, el impacto de El Niño en las instalaciones donde se realizaron obras de mitigación fue mucho menor que en aquellas donde no existieron programas ${ }^{(62)}$

Este mismo reporte indica que países como Ecuador y Perú, tuvieron la necesidad de dar respuesta en forma continua a las necesidades de la población, y la estructura nacional respondió en forma oportuna, desarrollando sistemas de búsqueda de información y comunicación por medio de Internet. Este medio permitió buscar información en redes y organismos científicos de meteorología (mundiales y regionales), intercambiar información entre países y compartir planes de contingencia e información sobre acciones de respuesta del sector Salud.

En la región, Cuba es considerada un modelo, debido a que cuenta con una política global de gestión de riesgos de desastres naturales que puede mejorar la continuidad de la asistencia para el desarrollo y reducir la ayuda humanitaria necesaria. Los planes preventivos ante desastres han sido una manera viable de ayudar a las personas, reduciendo los costos de emergencias, recuperación y reconstrucción ${ }^{(63)}$.

Otra experiencia exitosa en la implementación de estrategias efectivas para mitigar el daño de los desastres naturales en la salud y desarrollo humano es la de Bangladesh. Estas intervenciones se clasificaron de acuerdo a la fase del desastre: Las intervenciones en la fase predesastre incluyeron: creación de centros de acogida cercanos; difusión de la información, y la movilización de las familias en situaciones de riesgo. Durante la fase de desastre se brindó especial atención a la salud en general, mediante mejoras en cobertura de vacunación, sistemas de atención sanitaria, nutrición, agua y saneamiento, disponibilidad de medicamentos a bajo costo y tratamiento intradomiciliario de diarreas. Finalmente, después del desastre se priorizaron: distribución de agua segura y alimentos, cuidados sanitarios y otros servicios básicos, con estrategias focalizadas en personas con mayores necesidades ${ }^{(64)}$.
Otra revisión de la literatura brindó recomendaciones de planificación y preparación para prevenir enfermedades transmisibles durante desastres en dos fases: antes del desastre y después del desastre. Los planes de respuesta de emergencia antes de los desastres incluían: capacitación en identificación y manejo de las enfermedades específicas potencialmente amenazantes; preparación de los equipos, insumos y materiales necesarios; contar con insumos y materiales locales alternativos para el diagnóstico y tratamiento; y medidas de salud ambiental para controlar brotes epidémicos. La respuesta de emergencia para controlar las enfermedades transmisibles después del desastre incluían: atención médica de emergencia; provisión de refugios y planes de ubicación; agua y saneamiento; preparación de alimentos seguros; nutrición; manejo de casos; e insumos médicos y control de vectores. Además, la educación sanitaria y la entrega de servicios de salud son puntos críticos ${ }^{(65)}$

\section{DAÑOS A LA SALUD VINCULADOS AL FEN}

\section{ENFERMEDADES TRANSMITIDAS POR VECTORES}

Un estudio analizó la relación entre los eventos FEN y las epidemias de malaria en países de América Latina (Ecuador, Guyana Francesa, Surinam, Colombia, Guyana, Perú y Venezuela), encontrando relaciones significativas en los cuatro últimos países. Los autores demostraron que las inundaciones generan epidemias de malaria en las costas áridas del norte de Perú, mientras que las sequías favorecen el desarrollo de epidemias en Colombia y Guayana ${ }^{(32)}$.

Una revisión sistemática (Tabla 3 ) sobre efectos en salud de las sequías ${ }^{(66)}$, también incluyó una revisión sobre los efectos en la migración, encontrando estudios que asocian epidemias de malaria en zonas donde las poblaciones migraron debido a sequías en Etiopía. Si bien es cierto que existen varios informes de problemas de salud inducidos por la sequía relacionada a la migración, aún es poca la evidencia sobre las intervenciones efectivas para enfrentar estos problemas.

Una revision de 24 revisiones sistemáticas relacionadas a la prevención de enfermedades transmitidas por vectores estuvieron enfocados, sobre todo, en quimioprofilaxis, inmunizaciones y medidas de control del vector. Para cinco de las revisiones sobre dengue, la eficacia de las intervenciones se calculó como la tasa de índices entomológicos entre los grupos intervención y control ${ }^{67)}$.

\section{ENFERMEDADES TRANSMITIDAS POR EL AGUA}

Las Iluvias torrenciales son, generalmente, un factor importante en la contaminación del agua. Algunas 
causas de diarrea que están relacionadas a consumo de agua contaminada e inundaciones incluyen cólera, tifoidea y shigelosis.

En el caso de Ecuador, la sensibilización y la educación de la comunidad, la detección oportuna y el tratamiento adecuado de los casos, la rehidratación sistemática de los pacientes, la ayuda internacional y nacional para mejorar la vigilancia y fortalecer la capacidad resolutiva de los laboratorios, tanto como la desinfección del agua, contribuyeron a reducir el impacto de una nueva epidemia de cólera, en términos de letalidad como de incidencia, en el año 1998 durante el FEN ${ }^{(42) .}$

Una revisión de revisiones sistemáticas ${ }^{(67)}$ (Tabla 3) que evaluaron el impacto de intervenciones de tratamiento intradomiciliario de agua en diarrea autorreportada concluyó que la evidencia a favor de estas intervenciones es divergente entre los estudios. Dentro de este grupo de intervenciones los filtros de cerámica fueron los que mostraron mayor efectividad, pero la calidad de información científica de sustento fue baja.

\section{MALNUTRICIÓN}

El mayor impacto en salud pública de las sequías es en el estado nutricional con implicancias en morbilidad y mortalidad. Sin embargo, estos impactos son generalmente indirectos y complejos, asociados a alteraciones del ecosistema, reduciendo el suministro de alimentos que, a su vez, reduce de cantidad y/o calidad de la ingesta de nutrientes, que conlleva a una mayor susceptibilidad a enfermar, incrementando el riesgo de mortalidad. Los efectos negativos de las sequías pueden ser minimizados por intervenciones. Por ejemplo, un estudio en Lesotho ${ }^{(68)}$ encontró un efecto protector cuando se distribuye ayuda alimentaria; otro estudio en Kenia ${ }^{(69)}$ observó que los infantes (pero no los escolares) estuvieron "protegidos" de la sequía, aparentemente por cambios en comportamientos de la familia y distribución de alimentos: los cuidadores perdieron peso pero la ingesta de alimentos de los infantes no cambió y continuaron creciendo.

\section{ESTRÉS TÉRMICO}

Durante el verano de 1997, como consecuencia del FEN, investigadores peruanos describieron el cuadro clínico y la evolución de los casos de pacientes lactantes que acudieron con cuadros febriles durante los días de mayor temperatura y que se acompañaron de cuadros clínicos sugerentes de golpe de calor. Una vez analizados los datos, concluyeron que en situaciones ambientales adversas, debe considerarse el calor ambiental como un factor importante en el desarrollo de la hipertermia, con el propósito de brindar un manejo oportuno a los afectados en el menor tiempo posible ${ }^{(70)}$.

La revisión de revisiones sistemáticas (Tabla 3) analizó dos revisiones sistemáticas relacionadas al estrés térmico, encontrando que la principal intervención de salud pública para el estrés térmico son los planes de acción de salud térmica, a pesar de no encontrar evidencia sólida que apoye la información pública frente a las olas de calor en reducción de mortalidad (67).

\section{SEQUIAS}

EI Niño - Oscilación Sur también está asociado a sequía, donde las zonas más afectadas son el sureste de Asia, India, Australia; sureste de África, Amazonía y noreste de Brasil. Generalmente, los desastres desencadenados por las sequías son dos veces más frecuentes en el año siguiente al inicio del FEN que durante otros años ${ }^{(66)}$.

Una revisión sistemática sobre los efectos en la salud de las sequías (Tabla 3 ) concluye que las medidas de prevención frente a las sequías son clave y deberían incorporar el amplio rango de impactos que las sequías pueden tener en la salud humana, con énfasis en la prevención, preparación y mitigación, en lugar que enfocarse únicamente en el manejo de la crisis de los efectos ${ }^{(66)}$. Los autores recomiendan aplicar las estrategias de adaptación para fortalecer el sector salud frente a los desastres naturales asociados al cambio climático, descritas en el Reporte de Cambio Climático del 2007 (71): Planes de acción calor-salud; servicios médicos de emergencia; mejora de la vigilancia y control de las enfermedades sensibles al clima: agua segura y mejora del saneamiento.

\section{ENFERMEDADES ASOCIADAS A INUNDACIONES}

La experiencia de Bangladesh en la implementación de estrategias efectivas para mitigar el daño de los desastres naturales en la salud y desarrollo humano fue analizada en la serie de Lancet, donde destacaron que la detección temprana, la alerta y la respuesta apropiada de los ciudadanos a esas alertas, han probado ser efectivas en la reducción de las muertes por inundaciones. Los avances en ciencia y tecnología (mejores radares, satélites y sistemas de procesamiento de información), sirenas y el uso público de canales dedicados al clima han mejorado la oportunidad de advertencias en las inundaciones ${ }^{(64)}$.

Un grupo de investigadores de Tyndall Centre for Climate Research elaboraron una revisión estratégica de la literatura ${ }^{(72)}$, analizando los mecanismos para enfrentar a los riesgos de salud pública frente a las inundaciones. 
La revisión de la literatura ha encontrado diversos mecanismos y estrategias en relación a la protección de la salud en el hogar y la comunidad, la educación en salud e higiene, las prácticas de alerta y evacuación, la vigilancia y control de enfermedades, la prestación de asistencia sanitaria, la protección de la infraestructura del sistema de salud así como la protección y suministro de agua y saneamiento. Estas respuestas pueden darse antes, durante y después de las inundaciones.

\section{PROBLEMAS DE SALUD MENTAL}

Una revisión sistemática (Tabla 3) analizó los efectos globales de las inundaciones sobre la salud humana, e incluyó una sección especial sobre la salud mental, concluyendo que los problemas de salud mental fueron causados directamente por la experiencia de estar en una inundación, o indirectamente durante el proceso de reconstrucción. Sin embargo, los autores refieren que han encontrado limitada evidencia sobre intervenciones en salud pública (como la necesidad de medidas para reducir la propagación de enfermedades infecciosas, manejo de impactos en la salud mental, enfocado en grupos vulnerables), y no encontraron estudios sobre la efectividad de medidas de salud pública, incluyendo sistemas de alerta temprana ${ }^{(47)}$.

Otra revisión sistemática sobre los efectos de las sequías en la salud mental (Tabla 3) concluyó que la evidencia disponible sugiere que las sequías han tenido impacto negativo en la salud mental de las poblaciones de comunidades rurales cuyas vidas, generalmente, dependen de las lluvias. Los autores señalan que las intervenciones esenciales para enfrentar las sequías (y por tanto, sus efectos en la salud humana) son las alertas tempranas y los sistemas de vigilancia (para advertir a la gente sobre las posibles amenazas en su vida cotidiana, y para crear un registro histórico que evalúe las condiciones cambiantes); igualmente, otra intervención efectiva es la identificación de grupos vulnerables, regiones y sectores con mayor riesgo de los efectos de la sequía ${ }^{(66)}$.

Tabla 3. Revisiones sistemáticas de intervenciones en salud pública frente a desastres naturales

\begin{tabular}{|c|c|c|c|c|}
\hline Autor & $\begin{array}{l}\text { Tipo de } \\
\text { estudio }\end{array}$ & Intervención & $\begin{array}{l}\text { Número de } \\
\text { estudios } \\
\text { incluidos }\end{array}$ & Resultados principales \\
\hline $\begin{array}{l}\text { Kovats et al. } \\
\text { 2003. Lancet }\end{array}$ & $\begin{array}{l}\text { Revisión } \\
\text { Sistemática }\end{array}$ & $\begin{array}{l}\text { Efectividad del método } \\
\text { de Estudios de series } \\
\text { temporales para predecir } \\
\text { el fenómeno de El Niño }\end{array}$ & $\begin{array}{l}21 \text { estudios en } \\
18 \text { países }\end{array}$ & $\begin{array}{l}\text { La evidencia más sólida se encontró cuando el análisis } \\
\text { utilizó series temporales largas que incorporaban } \\
\text { más de un evento ENSO y donde hay congregación } \\
\text { geográfica de datos disponibles }\end{array}$ \\
\hline $\begin{array}{l}\text { Bouzid et al. } \\
2013\end{array}$ & $\begin{array}{l}\text { Revisión de } \\
\text { Revisiones } \\
\text { Sistemáticas }\end{array}$ & $\begin{array}{l}\text { Efectividad de } \\
\text { intervenciones en salud } \\
\text { pública para reducir el } \\
\text { impacto en la salud del } \\
\text { cambio climático }\end{array}$ & $\begin{array}{l}33 \text { revisiones } \\
\text { sistemáticas }\end{array}$ & $\begin{array}{l}\text { Las intervenciones ambientales fueron las que } \\
\text { presentaron la evidencia más débil, como el tratamiento } \\
\text { intradomiciliario de agua para consumo humano } \\
\text { que presentó evidencia y resultados divergentes. } \\
\text { Por otro lado, la revisión señala que la vacunación } \\
\text { y la quimioprofilaxis para prevenir enfermedades } \\
\text { transmitidas por vectores presentan la evidencia más } \\
\text { sólida, a pesar de estar limitadas a enfermedades } \\
\text { infecciosas aisladas }\end{array}$ \\
\hline $\begin{array}{l}\text { Few et al. } \\
2004\end{array}$ & $\begin{array}{l}\text { Revisión } \\
\text { estratégica } \\
\text { de la } \\
\text { literatura }\end{array}$ & $\begin{array}{l}\text { Analizar los mecanismos } \\
\text { para enfrentar los riesgos } \\
\text { de salud pública frente a } \\
\text { las inundaciones }\end{array}$ & $\begin{array}{l}35 \text { estudios en } \\
15 \text { países }\end{array}$ & $\begin{array}{l}\text { La evidencia encontró que las posibilidades de } \\
\text { respuesta efectiva frente a los riesgos sanitarios } \\
\text { se incrementan con información confiable de } \\
\text { las necesidades de salud de la población y las } \\
\text { necesidades de rehabilitación de sistemas de salud, } \\
\text { agua y saneamiento afectados por las inundaciones, } \\
\text { así como la comunicación de la información en forma } \\
\text { oportuna a la población. Asimismo, la planificación de } \\
\text { avanzada aparece fuertemente como la clave para } \\
\text { la implementación exitosa de la educación sanitaria, } \\
\text { alerta y evacuación, entrega de cuidado de salud en } \\
\text { emergencia, protección de la infraestructura, entre } \\
\text { otros }\end{array}$ \\
\hline $\begin{array}{l}\text { Ahern et al. } \\
2005\end{array}$ & $\begin{array}{l}\text { Revisión } \\
\text { sistemática }\end{array}$ & $\begin{array}{l}\text { Impactos en la salud de } \\
\text { las inundaciones }\end{array}$ & 36 estudios & $\begin{array}{l}\text { Encontraron limitada evidencia sobre intervenciones } \\
\text { en salud pública, y no encontraron estudios sobre la } \\
\text { efectividad de medidas de salud pública, incluyendo } \\
\text { sistemas de alerta temprana }\end{array}$ \\
\hline $\begin{array}{l}\text { Stanke et al. } \\
2013\end{array}$ & $\begin{array}{l}\text { Revisión } \\
\text { sistemática }\end{array}$ & $\begin{array}{l}\text { Evidencia de los efectos } \\
\text { en la salud pública de las } \\
\text { sequías }\end{array}$ & 87 estudios & $\begin{array}{l}\text { Una intervención esencial son las alertas tempranas y } \\
\text { los sistemas de vigilancia de la sequía (para advertir } \\
\text { a la gente sobre las posibles amenazas en su vida } \\
\text { cotidiana, y para crear un registro histórico que evalúe } \\
\text { las condiciones cambiantes); otra intervención efectiva } \\
\text { es la identificación de grupos vulnerables, regiones y } \\
\text { sectores con mayor riesgo de los efectos de la sequía }\end{array}$ \\
\hline
\end{tabular}




\section{VULNERABILIDAD DE INFRAESTRUCTURA FÍSICA DE LOS SERVICIOS DE SALUD}

En lo relacionado a la vulnerabilidad en el diseño de los establecimientos de salud frente a desastres naturales, la OPS ${ }^{(73)}$ señala que la mayor parte de las pérdidas en infraestructura de salud se deben a su ubicación en zonas vulnerables, diseños inadecuados o falta de mantenimiento de los establecimientos. Habitualmente los planes de protección o de mitigación de instalaciones de salud frente a desastres prevén la seguridad de las personas y no tanto de las instalaciones o de la operatividad.

Un artículo ${ }^{(74)}$ calculó el impacto económico del fenómeno de El Niño 1998 en los servicios de salud de Zorritos en Tumbes y concluyó que este impacto fue de S/ 567 457, desagregados en: costos sanitarios (reparación temporal de establecimientos de salud: S/ 13,000; atención de enfermedades trazadoras: S/ 136 108; otras atenciones: S/ 246 704) y costos no sanitarios (S/ 171 645). Este estudio muestra que el principal costo se asocia con la malaria, las enfermedades diarreicas agudas y las infecciones respiratorias agudas, con una participación del $42 \%$ del costo estimado.

Sobre la vulnerabilidad en el diseño de los establecimientos de salud frente a desastres naturales, la OPS (73) recomienda que las áreas esenciales de los hospitales sean construidas con el tercer nivel de protección, y que el conjunto de todo nuevo establecimiento de salud sea construido, por lo menos, con el primer nivel de protección; todo esto para disponer que los nuevos establecimientos de salud sean capaces de garantizar también los objetivos de seguridad de la infraestructura y de la operación.

\section{POLÍTICAS A LARGO PLAZO}

Un objetivo común a muchos países es comprender bien cómo la variabilidad climática y el cambio climático afectan los riesgos para la salud ahora y en el futuro, de tal manera que les permita elaborar políticas y programas que protejan la salud pública. La habilidad de un país o comunidad para identificar e implementar políticas y programas de adaptación efectivos, depende de muchos factores: autoridades y comunidad civil debidamente informadas, autoridades motivadas para la acción, autoridades con conocimiento de la magnitud de los posibles riesgos y de las opciones posibles, recursos disponibles e instituciones principales estructuradas ${ }^{(75)}$.

Un estudio que analizó las lecciones aprendidas para implementar intervenciones de planificación de políticas de salud pública para enfrentar cuatro enfermedades infecciosas (malaria, dengue, chikungunya y cólera), dirigido a tomadores de decisiones de Francia en particular, y de otros países en general, incluyó las siguientes recomendaciones: revisión de los planes operativos de los programas de salud pública, implementación de una estrategia más activa e integrativa de gestión de programas, desarrollo de potenciales herramientas sólidas y resistentes, reconciliación entre las diferentes agendas de las partes interesadas, desarrollo de políticas articuladas de salud y ambiente, promoción y apoyo para la investigación de patógenos y sus hábitats ${ }^{(76)}$.

Actualmente, se reconoce que existen puntos críticos del cambio climático (en donde se enmarcan los ciclos del fenómeno $E$ Niño) que vienen afectando a la salud humana, y en especial a las poblaciones de menores ingresos. Algunos autores han realizado revisiones de reportes publicados por organizaciones internacionales que investigan el cambio climático (77) y luego de analizar puntos críticos recomiendan a los gobiernos implementar medidas de adaptación como: programas de vacunación, vigilancia epidemiológica, monitoreo y uso de tecnologías protectoras (pesticidas, filtración y tratamiento de agua, pronósticos de tiempo, etc).

Adicionalmente, la Comisión de Salud y Cambio Climático de la revista Lancet, constituida en el $2015^{(61)}$ por un grupo multidisciplinario de científicos internacionales, recomienda entre otras cosas, que los países deben:

a) Invertir en investigaciones sobre el cambio climático y salud pública, monitoreo y vigilancia para garantizar una mejor comprensión de las vulnerabilidades, necesidades de adaptación y los potenciales beneficios de la mitigación del cambio climático en el nivel local y nacional.

b) Ampliar el financiamiento de los sistemas de salud resilientes al clima en todo el mundo. Lo cual implica preparar y mejorar la infraestructura y la logística de los mismos.

c) Brindar soporte a la cuantificación exacta de la carga de la enfermedad evitada, reducción de los costos de atención de la salud y la mejora de la productividad económica asociada a la mitigación del cambio climático.

La Corporación Andina de Fomento ${ }^{(78)}$ identificó algunas lecciones aprendidas orientadas a la reducción de la vulnerabilidad del sector Salud del Perú frente al FEN de los años 1997-1998, que constituyen una plataforma para las actuaciones futuras:

a) Confiabilidad de los pronósticos: ante la evidencia de la proximidad de un nuevo evento, se debe contar con un adecuado conocimiento sobre las amenazas, que proviene de diferentes instituciones responsables, para lograr una mejor predicción de los 
acontecimientos, profundizar y realizar simulaciones de los posibles impactos sobre el sector.

b) La respuesta del sector Salud para con los damnificados, el tratamiento de enfermedades infecciosas y otros daños somáticos, fueron realizados con efectividad; sin embargo, otras intervenciones como las dirigidas a información y comunicación, mitigación de impactos sobre la salud mental de los damnificados, riesgo nutricional infantil y de los ancianos, no tuvieron el mismo nivel de atención y de preocupación.

c) Debido a la naturaleza compleja de este tipo de fenómenos, la respuesta debe ser multisectorial y llevarse a cabo de manera coordinada, a fin de reducir el impacto de una manera más efectiva.

Es importante recordar que los efectos asociados a desastres naturales afectan a todos los grupos etarios, pero las políticas públicas deben tener especial cuidado en la focalización de los grupos vulnerables y seguir las recomendaciones de UNICEF ${ }^{(79)}$, para la atención de estos grupos vulnerables.

\section{CONCLUSIONES}

De acuerdo con la literatura científica existen intervenciones efectivas para enfrentar los daños a la salud producidos por el cambio climático, los cuales implican también los eventos producidos por el FEN. En estas, se incluyen intervenciones ambientales de control de vectores, quimioprofilaxis, vacunación, y tratamiento de agua intradomiciliario y comunitario. Por otro lado, las intervenciones ambientales, como el tratamiento de agua intradomiciliario para consumo humano, presentan evidencia y resultados divergentes.

Las posibilidades de respuesta efectiva frente a los riesgos sanitarios se incrementan con información confiable de las necesidades de salud de la población y las necesidades de rehabilitación de sistemas de salud, agua y saneamiento afectados por las inundaciones, así como la comunicación de la información en forma oportuna a la población.

Se evidencia también que una intervención esencial para enfrentar las sequías y, por tanto, sus efectos en la salud humana, son las alertas tempranas y los sistemas de vigilancia de la sequía (para advertir a la gente sobre las posibles amenazas en su vida cotidiana, y para crear un registro histórico que evalúe las condiciones cambiantes); igualmente, otra intervención efectiva es la identificación de grupos vulnerables, regiones y sectores con mayor riesgo de los efectos de la sequía.

Si bien la evidencia se basa principalmente en revisiones sistemáticas, es necesario considerar el contexto de cada país, antes de plantear recomendaciones en salud pública basada en evidencia.

Así mismo, la disponibilidad de agua segura para las poblaciones es una meta esencial, sobre todo en situaciones de desastres, existiendo intervenciones simples, aceptables y de bajo costo para el tratamiento intradomiciliario de agua ${ }^{(80)}$. Es necesario considerar que no siempre es posible encontrar evidencia fuerte que sustente intervenciones en salud pública, sobre todo las que se aplican a poblaciones pobres. Esto puede suceder, entre otros motivos, por la falta de financiamiento de estudios de alta calidad metodológica ${ }^{\left({ }^{(81)}\right.}$. Por lo tanto, es necesario considerar otros factores como la necesidad de la población, la equidad y la implementabilidad para evaluar las intervenciones antes de la aplicación rígida de las reglas de la evidencia científica.

Por último, se sugiere desarrollar investigaciones, monitoreo y vigilancia del FEN y su implicancia en la salud pública, para garantizar una mejor comprensión de las vulnerabilidades, necesidades de adaptación de medidas a nivel local, regional y nacional.

Contribuciones de autoría: GH, CB, CVM, ELG y WR participaron en la concepción del trabajo, todos los autores participaron en la redacción del manuscrito, en la revisión crítica del manuscrito y en la aprobación final del manuscrito.

Fuente de financiamiento: el financiamiento se obtuvo del Instituto Nacional de Salud. Lima, Perú

Conflictos de interés: El presente manuscrito no necesariamente representa una opinión oficial del Departamento de Medicina Preventiva y Bioestadistica - Uniformed Services University of the Health Sciences, la Universidad San Martín de Porres, o del Instituto de Investigaciones Clínicas de la UNMSM.

\section{REFERENCIAS BIBLIOGRÁFICAS}

1. Voituriez B. Los caprichos del Océano. Efectos sobre el clima y los recursos vivos. Barcelona: UNESCO; 2003.

2. Zambrano E. El fenómeno de "El Niño" y la oscilación del sur (ENSO). Acta Oceanográfica del Pacífico INOCAREcuador. 1986;3(1):195-203.
3. Ramos W. Impacto del fenómeno El Niño en el país: ¿Qué podemos esperar para el año 2016? Bol Epidemiol (Lima). 2015;24(35):694-6.

4. Kovats RS, Bouma MJ, Hajat S, Worrall E, Haines A. El Niño and health. Lancet.
2003;362(9394):1481-9. doi: 10.1016/ S0140-6736(03)14695-8.

5. Cane MA. The evolution of El Niño, past and future. Earth Planet Sci Letters 2005;230:227-40.

6. Servicio Nacional de Meteorología e Hidrología (SENAMHI). El fenómeno 
El Niño en el Perú. Lima: SENAMHI; 2014.

7. Wang C, Deser C; Yu J, Dinezio P, Clement A. El Niño-Southern Oscillation (ENSO): A review. In: Glymn P, Manzello D, Enochs I, editors. Coral Reefs of the Eastern Pacific. Berlín: Springer Science Publisher; 2012.

8. Bouma MJ, Kovats RS, Goubet SA, Cox JS, Haines A. Global assessment of El Niño's disaster burden. Lancet. 1997;350:1435-8. doi: http://dx.doi. org/10.1016/S0140-6736(97)04509-1.

9. Dilley M, Heyman BN. ENSO and disaster: droughts, floods and El Niño/ Southern Oscillation warm events. Disasters. 1995;19(3):181-93.

10. Comisión Económica para América Latina y El Caribe (CEPAL). El impacto de los desastres naturales en el desarrollo: documento metodológico básico para estudios nacionales de caso. Santiago de Chile: CEPAL; 2005.

11. Secretaría de Salud de México. Programa De Acción Específico 2007-2012. Urgencias epidemiológicas y desastres. México, D.F.: Secretaría de Salud; 2008.

12. Federación Internacional de Sociedades de la Cruz Roja y de la Media Luna Roja. Informe mundial sobre desastres 2010. Ginebra: IFRC; 2010.

13. Kovats RS. El Nino and human health. Bull World Health Organ. 2000;78(9):1127-35.

14. Ramos W. Vigilancia con posterioridad a desastres: una necesidad en el contexto sanitario y climático actual. Bol Epidemiol (Lima); 2015; 24(50):947-8.

15. Rocha A. El meganiño 1982-83, "la madre de todos los niños”. Lima: IMEFEN/UNI; 2007.

16. Franco E. El niño en América Latina. Desastres y sociedad. 1998;6(9):3-6.

17. Consejo Económico y Social de las Naciones Unidas, Comisión Económica para América Latina (CEPAL). Los desastres naturales de 1982-193 en Bolivia, Ecuador y Perú. Nueva York: ONU/CEPAL; 1983.

18. Lavado-Casimiro W, Espinoza JC. Impactos de El niño y La niña en las lluvias del Perú (1965-2007). Rev Bras Metereol. 2014;29(2):11-82.

19. Pan American Health Organization (PHAO). Protecting new health facilities from natural disasters: Guidelines for the promotion of disaster mitigation. Washington, D.C: PAHO/World Bank; 2003.
20. Huang X, Clements AC, Williams G, Devine G, Tong S, Hu W.El Niño-Southern Oscillation, local weather and occurrences of dengue virus serotypes. Sci Rep. 2015;5:16806. doi: 10.1038/srep16806.

21. Chretien JP, Anyamba A, Small J, Britch S, Sanchez JL, Halbach AC, et al. Global Climate Anomalies and Potential Infectious Disease Risks: 2014-2015. PLOS Curr. 2015;7: pii: ecurrents.outbreaks.95fbc4a8fb4695e049baabfc2fc8289f. doi: 10.1371/ currents.outbreaks. $95 \mathrm{fbc} 4 \mathrm{a} 8 \mathrm{fb} 4695 \mathrm{e}$ 049baabfc2fc8289f.

22. Stewart-Ibarra AM, Lowe R. Climate and non-climate drivers of dengue epidemics in southern coastal ecuador. Am J Trop Med Hyg. 2013;88(5):971-81. doi: 10.4269/ajtmh.12-0478.

23. Cazelles B, Chavez M, McMichael AJ, Hales S. Nonstationary influence of El Niño on the synchronous dengue epidemics in Thailand. PLoS Med. 2005;2(4):e106. doi: 10.1371/journal.pmed.0020106.

24. Earnest A, Tan SB, Wilder-Smith A. Meteorological factors and El Niño Southern Oscillation are independently associated with dengue infections. Epidemiol Infect. 2012;140(7):1244-51. doi: 10.1017/S095026881100183X.

25. Colón-González FJ, Lake IR, Bentham G. Climate variability and dengue fever in warm and humid Mexico. Am J Trop Med Hyg. 2011;84(5):757-63. doi: 10.4269/ ajtmh.2011.10-0609.

26. Johansson MA, Cummings DA, Glass GE. Multiyear climate variability and dengue-El Niño southern oscillation, weather, and dengue incidence in Puerto Rico, Mexico, and Thailand: a longitudinal data analysis. PLoS Med. 2009;6(11):e1000168. doi: 10.1371/journal.pmed.1000168.

27. Brunkard JM, Cifuentes E, Rothenberg SJ. Assessing the roles of temperature, precipitation, and ENSO in dengue reemergence on the Texas-Mexico border region. Salud Publica Mex. 2008;50(3):227-34.

28. Gagnon AS, Bush ABG, Smoyer-Tomic KE. Dengue epidemics and the El Niño Southern Oscillation. Clim Res. 2001;19:35-43.

29. Delgado-Petrocelli L, Córdova K, Camardiel A, Aguilar VH, Hernández D, Ramos S. Analysis of the El Niño/La Niña Southern Oscillation variability and malaria in the Estado Sucre, Venezuela. Geospat Health. 2012;6(3):S51-57.

30. Zubair L, Galappaththy GN, Yang H, Chandimala J, Yahiya Z, Amerasinghe P, et al. Epochal changes in the association between malaria epidemics and El Niño in Sri Lanka. Malar J. 2008;7:140. doi: 10.1186/1475-2875-7-140.

31. Mantilla G, Oliveros H, Barnston AG. The role of ENSO in understanding changes in Colombia's annual malaria burden by region, 1960-2006. Malar J. 2009;8:6. doi: 10.1186/1475-2875-8-6.

32. Gagnon AS, Smoyer-Tomic KE, Bush AB. The Niño Southern Oscillation and malaria epidemics in South America. Int J Biometeorol. 2002;46(2):81-9.

33. Cardenas R, Sandoval CM, RodríguezMorales AJ, Franco-Paredes C. Impact of climate variability in the occurrence of leishmaniasis in northeastern Colombia. Am J Trop Med Hyg. 2006;75(2):273-7.

34. Chinga-Alayo E, Huarcaya E, Nasarre C, del Aguila R, Llanos-Cuentas A. The influence of climate on the epidemiology of bartonellosis in Ancash, Peru. Trans R Soc Trop Med Hyg. 2004;98(2):116-24.

35. Hjelle B, Glass GE. Outbreak of hantavirus infection in the Four Corners region of the United States in the wake of the 1997-1998 El Ninosouthern oscillation. J Infect Dis. 2000;181(5):1569-73.

36. Weinberger D, Baroux N, Grangeon JP, Ko AI, Goarant C. El Niño Southern Oscillation and leptospirosis outbreaks in New Caledonia. PLoS Negl Trop Dis. 2014;8(4):e2798. doi: 10.1371/journal. pntd.0002798.

37. Organización Panamericana de la Salud (OPS). Crónicas de desastres. Fenómeno El Niño 1997-1998. Washington, D.C.: OPS; 2000.

38. Cash BA, Rodó X, Emch M, Yunus M, Faruque AS, Pascual M. Cholera and shigellosis: different epidemiology but similar responses to climate variability. PLoS One. 2014;9(9):e107223. doi: 10.1371/journal.pone.0107223.

39. Hashizume M, Chaves LF, Faruque AS, Yunus M, Streatfield K, Moji K. A differential effect of Indian ocean dipole and El Niño on cholera dynamics in Bangladesh. PLoS One. 2013;8(3):e60001. doi: 10.1371/ journal.pone.0060001.

40. Ohtomo K, Kobayashi N, Sumi A, Ohtomo N. Relationship of cholera incidence to El Niño and solar activity elucidated by time-series analysis. Epidemiol Infect. 2010;138(1):99-107. doi: $10.1017 /$ S0950268809990203. 
41. Rodo X, Pascual M, Fuchs G, Faruque AS. ENSO and cholera: a nonstationary link related to climate change? Proc Natl Acad Sci USA. 2002;99(20):12901-6.

42. Gabastou JM, Pesantes C, Escalante S, Narvaez Y, Vela E, García L, et al. Características de la epidemia de cólera de 1998 en Ecuador, durante el fenómeno de El Niño". Rev Panam Salud Publica. 2002;12(3):157-64.

43. Oluwole OS. Climate Regimes, El Niño-Southern Oscillation, and Meningococcal Meningitis Epidemics. Front Public Health. 2015;3:187. doi: 10.3389/fpubh.2015.00187.

44. Gutierrez EL, Galarza C, Ramos W, Mendoza M, Smith ME, Ortega-Loayza AG. Influence of climatic factors on the medical attentions of dermatologic diseases in a hospital of Lima, Peru. An Bras Dermatol. 2010;85(4):461-8.

45. Mazzarella A, Giuliacci A, Pregliasco F. Hypothesis on a posible role of El Nino in the occurrence of influenza pandemics. Theor Appl Climatol. 2011;105(1):65-9. doi: 10.1007/ s00704-010-0375-7.

46. Oluwole OS. Seasonal Influenza Epidemics and El Niños. Front Public Health. 2015;3:250. doi: 10.3389/ fpubh.2015.00250.

47. Ahern M, Kovats RS, Wilkinson P, Few R, Matthies F. Global Health Impacts of Floods: Epidemiologic Evidence. Epidemiol Rev. 2005;27:36-46.

48. McMillen C, North C, Mosley M, Smith E. Untangling the psychiatric comorbidity of posttraumatic stress disorder in a sample of flood survivors. Compr Psychiatry. 2002;43(6):478-85.

49. Russoniello CV, Skalko TK, O’Brien $\mathrm{K}$, McGhee SA, Bingham-Alexander D, Beatley J. Childhood posttraumatic stress disorder and efforts to cope after Hurricane Floyd. Behav Med. 2002;28(2):61-70.

50. Ginexi EM, Weihs K, Simmens SJ, Hoyt DR. Natural disaster and depression: a prospective investigation of reactions to the 1993 midwest floods. Am J Community Psychol. 2000;28(4):495518. doi: 10.1023/A:1005188515149.

51. Krug EG, Kresnow M, Peddicord JP, Dahlberg LL, Powell KE, Crosby $\mathrm{AE}$, et al. Retraction: suicide after natural disasters. $\mathrm{N}$ Eng $\mathrm{J}$ Med. 1999;340(2):148-9.

52. Tobin GA, Ollenburger JC. Predicting levels of postdisaster stress in adults following the 1993 floods in the upper midwest. Environ Behav. 1996;28(3):340-57. doi: 10.1177/0013916596283004.

53. Durkin MS, Khan N, Davidson LL, Zaman SS, Stein ZA. The effects of a natural disaster on child behaviour: evidence for posttraumatic stress. Am J Public Health. 1993;83(11):1549-53.

54. Phifer JF, Kaniasty KZ, Norris FH. The impact of natural disaster on the health of older adults: a multiwave prospective study. J Health Soc Behav 1988;29(1):65-78.

55. Logue JN, Melick ME, Struening EL. A study of health and mental health status following a major natural disaster. Res Community Ment Health. 1981;2:217-74.

56. Organización Panamericana de la Salud. Los desastres naturales y la protección de la salud. Publicación científica 575. Washington, D.C.: OPS; 2000.

57. Wiley LF. Mitigation/adaptation and health: health policymaking in the global response to climate change and implications for other upstream determinants. J Law Med Ethics. 2010;38(3):629-39. doi: 10.1111/j.1748-720X.2010.00516.x.

58. Bowen KJ, Ebi K, Friel S, McMichael AJ. A multi-layered governance framework for incorporating social science insights into adapting to the health impacts of climate change. Glob Health Action. 2013;6:21820. doi: 10.3402/gha.v6i0.21820.

59. Rivera C. Integrating climate change adaptation into disaster risk reduction in urban contexts: perceptions and practice. PLoS Curr. 2014;6: pii: ecurrents.dis.7bfa59d37f7f59abc238462d53fbb41f. doi: $\quad 10.1371 /$ currents.dis.7bfa59d37f7f59abc238462d53fbb41f.

60. Hammad KS, Arbon P, Gebbie K, Hutton A. Nursing in the emergency department (ED) during a disaster: a review of the current literature. Australas Emerg Nurs J. 2012;15(4):23544. doi: 10.1016/j.aenj.2012.10.005.

61. Watts N., Adger WN, Agmolucci P, Blackstock J, Byass P, Cai W, et al. Health and climate change: policy responses to protect public health. Lancet. 2015; 386(10006):1861-914. doi: 10.1016/ S0140-6736(15)60854-6.

62. Organización Panamericana de la Salud, Organización Mundial de la Salud. Repercusiones sanitarias del fenómeno El Niño. Washington, D.C.: OPS/ OMS; $1998.21 \mathrm{p}$.

63. Mas Bermejo P. Preparation and response in case of natural disaster:
Cuban programs and experienced. J Public Health Policy. 2006;27(1):13-21.

64. Cash RA, Halder SR, Husain $M$, Islam MS, Mallick FH, May MA, et al. Reducing the health effect of natural hazards in Bangladesh. Lancet. 2013;382(9910): 2094-103. doi: 10.1016/S0140-6736(13)61948-0.

65. Jafari N, Shahsanai A, Memarzadeh M, Loghmani A. Prevention of communicable diseases after disasters: A review. J Res Med Sci. 2011;16(7);956-62.

66. Stanke C, Kerac M, Prudhomme C, Medlock J, Murray V. Health effects of drought: a systematic review of the evidence. PLoS Curr. 2013;5: pii: ecurrents. dis.7a2cee9e980f9 1 ad 7697 b570bcc4b004. doi: 10.1371/currents. dis. 7 a 2cee9e980f9 1 ad 7697 b5 570 bcc4b004.

67. Bouzid M, Hoopera L, Hunter PR. The effectiveness of public health interventions to reduce the health impact of climate change: a systematic review of systematic reviews. PLoS One. 2013;8(4):e62041. doi: 10.1371/ journal.pone.0062041.

68. Renzaho A. Mortality, malnutrition and the humanitarian response to the food crises in Lesotho. Australas J Paramed [Internet]. 2012 [citado el 5 de enero de 2016];4(4):[15 pp.]. Disponible en: http://ajp.paramedics.org/index.php/ ajp/article/view/395

69. McDonald MA, Sigman M, Espinosa MP, Neumann CG. Impact of a Temporary Food Shortage on children and their mothers. Child Dev. 1994;65(2 Spec No):404-15.

70. Miranda J, Cabezas C, Maguiña C, Valdivia J. Hipertermia durante el fenómeno de El Niño, 1997-98. Rev Peru Med Exp Salud Publica. 2003;20(4):200-5.

71. Grupo Intergubernamental de Expertos sobre el Cambio Climático. Cambio Climático 2007: Informe de síntesis. Contribución de los grupos de trabajo I, II y III al Cuarto Informe de evaluación del Grupo Intergubernamental de Expertos sobre el Cambio Climático. Ginebra: IPCC; 2007.

72. Few R, Ahern M, Matthies F, Kovats S. Floods, health and climate change: a strategic review. Norwich: Tyndall Centre for Climate Change Research; 2004.

73. Pan American Health Organization. Guidelines for vulnerability reduction in the design of new health facilities. 
Washington, D.C.; PAHO/World Bank; 2004.

74. Bambarén C. Estimación del impacto económico de los desastres naturales en los servicios de salud. Seminario de tesis. Revista de la Facultad de Ciencias Económicas. 2000;5(15):201-15.

75. World Health Organization. Protecting health from climate change: vulnerability and adaptation assessment. Geneva: WHO; 2013.

76. Manigat R, Wallet F, André JC. From past to better public health programme planning for possible future global threats: case studies applied to infection control. Ann Ist Super Sanita. 2010;46(3):228-35. doi: 10.4415/ ANN_10_03_02.
77. Patz JA, Kovats RS. Hotspots in climate change and human health. BMJ. 2002;325(7372):1094-8. doi: http:// dx.doi.org/10.1136/bmj.325.7372.1094

78. Corporación Andina de Fomento. El Fenómeno El Niño 1997-1998. Memoria, Retos y Soluciones. Volumen V: Perú. Lima: CAF; 2000.

79. Yoko A, Goodman D, Parker D. Global climate change and child health: a review of pathways, impacts and measures to improve the evidence base. UNICEF. Discussion Paper. IDP No 2009-03. Florence: UNICEF Innocenti Research Centre; 2009.

80. Cruz R, Arévalo H, Chamorro F, Fernandez F. Efecto del uso de un método artesanal para el tratamiento de agua en comunidades rurales de la región San Martín, Perú. Rev Peru Med Exp Salud Publica. 2005; 22(2):117-22.

81. Rychetnik L, Frommer M, Hawe P, Shiell A. Criteria for evaluating evidence on public health interventions. J Epidemiol Community Health. 2002;56(2):119-27.

Correspondencia: Gisely Hijar Guerra Dirección: Calle Cápac Yupanqui 1400. Jesús María. Lima 11, Perí

Teléfono: (511) 748-1111 anexo 2113

Correoelectrónico:giselins@gmail.com

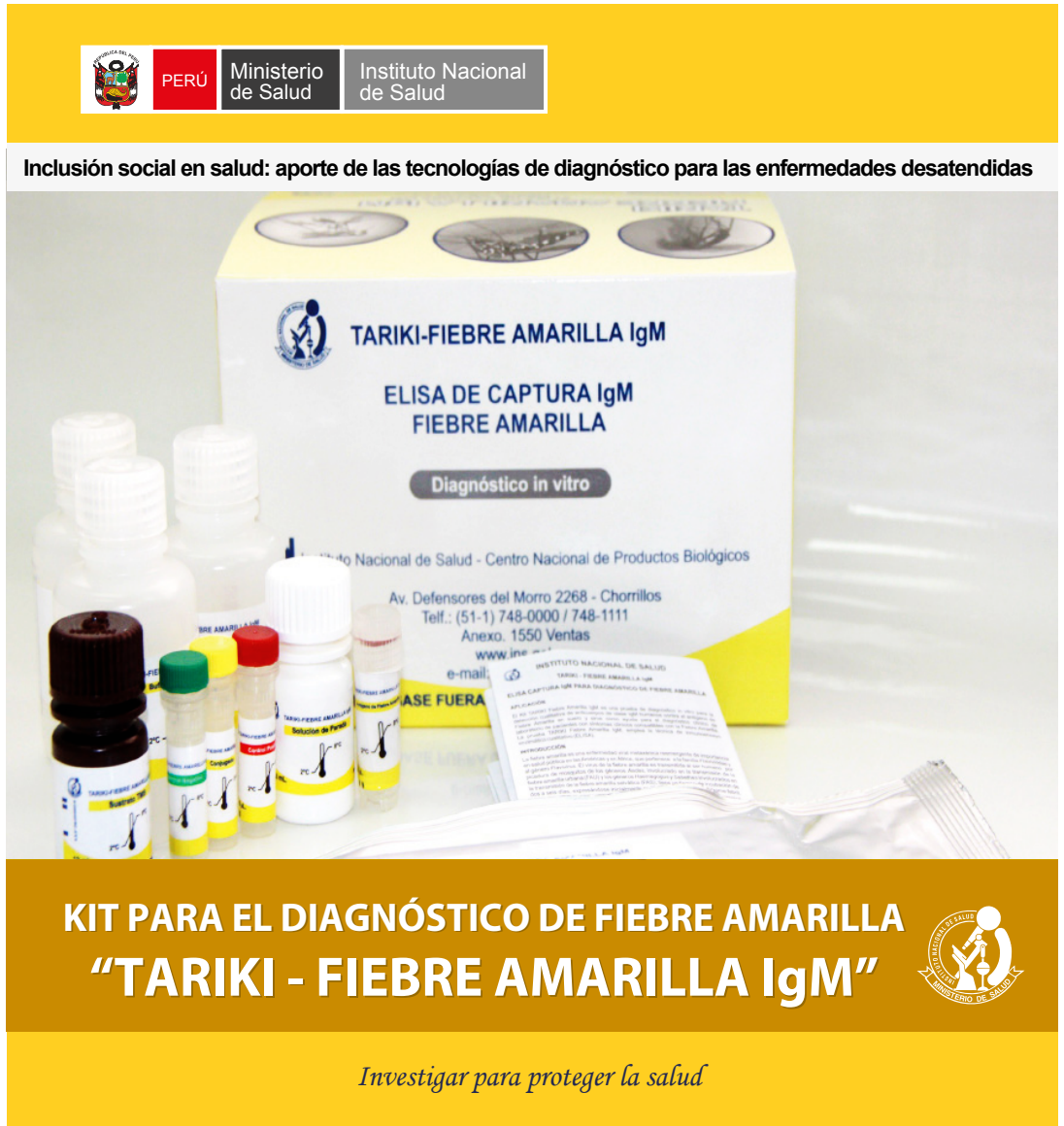

